\title{
Who should do interventional radiology?
}

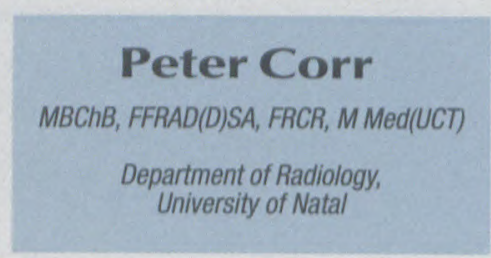

W ith rapid technical advances in interventional techniques over the last five years the role of the interventional radiologist in patient management is changing. The whole new speciality of "minimally invasive surgery" is developing led by the increased use of direct endoscopic techniques and is encroaching on many of the procedures that have been developed in part by the interventional radiologist. This is seen particularly in vascular and neurovascular interventional procedures where vascular surgeons, cardiologists and neurosurgeons are developing interest in endovascular therapy. The cross-over between radiology, cardiology and surgery has a number of important implications in training, skills development and accreditation both internationally and in the South African context.

Training in interventional radiology in North America usually entails a 12 month fellowship for general body intervention and 24 months for neurointervention. In the United Kingdom, the Royal College of Radiologists recommends a minimum of 12 months of full time post fellowship training in a recognised centre, of which there are 12 in the UK. In South Africa there is no training course although some departments have excellent registrar rotations in interventional radiology.

Development of interventional radiology is dependent on communication to both clinical colleagues and our own radiology colleagues of the benefits and applications of interventional techniques to patient management. From personal experience, convincing one's surgical colleagues of the advantages of interventional radiology can be a long and arduous process. The Society of Interventional Radiology has been formed locally and should be able to focus attention and improve education in this very important speciality.

Who should perform it? This is a very contentious question in Europe and North America; however in South Africa where we have a significant skills shortage, perhaps the most honest local answer is: "The most capable skilled specialist available". To develop interventional skills, it is very important to understand the disease process and clinical problems that the surgeon faces in patient management. Only by working in a team environment where there is a healthy collegial relationship between the radiologist and surgeon, will interventional radiology develop and prosper. The radiologist must be available 24 hours a day and must be prepared to be involved with management of the complications related to the procedures.

We need, as radiologists, to be aware of not only the rapid developments in this exciting field but also the added responsibilities to ensure that we do not lose our potential to contribute to patient care.

\section{The Editor}

